\title{
Editorial of the Open Journal on Modelling and Simulation
}

\author{
Alessandro Bazzi \\ IEIIT-CNR and WiLab @ DEI, University of Bologna, Bologna, Italy \\ Email: alessandro.bazzi@cnr.it
}

Received June 1, 2013; revised July 2, 2013; accepted July 9, 2013

Copyright (C) 2013 Alessandro Bazzi. This is an open access article distributed under the Creative Commons Attribution License, which permits unrestricted use, distribution, and reproduction in any medium, provided the original work is properly cited.

Dear Readers, it is an honor for me to introduce a new issue of the Open Journal on Modelling and Simulation (OJMSi). This is a young journal, at its third number, and yet observed hundreds of downloads and thousands of visits. In this first Editorial of OJMSi, I'll focus on a key topic for this journal, and something I am familiar with; I'll talk about simulation, sharing my several years of experience in simulators design and development, and remarking the primary role that it has in the modern science. My considerations arise from my field, i.e., wireless communications and networks, but I am confident that they remain valid for scientists working on any topic.

Instead of giving a definition of the term simulation (you can find dozens in the Internet), I will paraphrase something I heard at a seminar few years ago: measurements are those things that everyone believe to, except who performs them, whereas simulations are those things that no one believe to, except who performs them. I am not sure my personalization still reports exactly what the speaker intended, but anyone that had something to do with on-field measurements or with complex simulations surely understand and agrees with what I wrote.

Trying to group concepts in categories (as we engineers like so much), on field measurement, simulation, and analysis through models are the three methods that the scientists have to investigate a problem or validate a solution. Among these, simulations are by far the most powerful, but they are also those that face the highest scepticism.

It is out of doubts that performing measurements is the surest way to prove something: you can see it, so it is. However, measurements are not always possible: we cannot deploy a cellular network placing base stations to investigate the coverage granted to users. And even when it is possible, reading results and drawing conclusions is not always easy, since several effects are present that could sum up and become indistinguishable.

On the other hand, if an analytical framework perfectly models something, then results are absolutely unique and convincing, and the impact of each parameter can be easily checked. Any other scientist can take the equations and again obtain exactly the same conclusions. Modelling the reality is however a hard task. Assumptions must be made and approximations need to be introduced. Even spending nights with the mind in endless formulas, there is a point where the complexity becomes insurmountable. From that point on, only simulation can be used.

Simulation can be also used to validate analytical models, but I am not talking about this aspect; typically, in this case simulation is something simple, since it reproduces a scenario that can be approximated by equations. I am talking, instead, about simulation where a huge number of effects, rules and limitations jointly participate to a result. For example, I am talking about the study of wireless communications among vehicles in an urban scenario, where junctions and road rules heavily impact on mobility (yes, you discovered me, I am presently working on this); we can model a road segment, we can model some aspects of wireless communications, but we are not able to close everything into an analytical framework.

Simulation allows reproducing and studying very complex systems, but with the increase of complexity a problem grows and grows: how can we be sure that the simulation says the truth? The real point is that in most cases, to be fair, we cannot. We have no analysis or measurement to confirm results, because if we had there was no need for simulation. And we cannot hope that omniscient software will come to check our work, it will not. What we, as developers, must do is to validate the single pieces and test them under conditions that have known outputs. After that, we must resign ourselves that we will not be immune to errors, and that at least some minor errors are indeed surely hidden somewhere. It will always be a fight with bugs and debugs. But another really important point is what we, as users, must do: we must always perform simulations with deep knowledge 
of the tool. Simulators are based on models, and models always introduce approximations. Using a simulator without perfectly knowing the approximations it carries is like driving without knowing the road rules: risks are very high.

So, if you do not know how to go on with your analysis, because there are too many effects that must be ac- counted for, then move to simulation! And if you find a negative reviewer or a sceptic boss, saying that yours are only simulations, then send him the code and ask him to check if something is wrong!

Good simulations and good reading of this new issue of OJMSi! 